\title{
:QUÉ HA SALIDO MAL?: \\ HALLAZGOS SOBRE LA IMPLEMENTACIÓN DE \\ POLÍTICAS PÚBLICAS PARA LA TRANSPARENCIA A PARTIR DE UN CASO INSTRUMENTAL
}

\author{
Víctor S. Peña \\ El Colegio de Sonora, México \\ vpena@colson.edu.mx
}

\section{RESUMEN}

¿Qué sucede una vez que una decisión pública se lleva a la práctica? La respuesta a esta interrogante se encuentra en el campo de la implementación de la política pública, área estudiada de manera discontinua a lo largo del tiempo. Posterior a un recuento teórico, el presente artículo explora, a partir de un caso instrumental ubicado en un gobierno subnacional mexicano, que ha implicado llevar a la realidad una decisión legislativa relacionada con la transparencia. Entre los hallazgos destacan las situaciones de carácter político, los acuerdos entre grupos y por la concentración del poder en pocos actores por ejemplo, como elementos coadyuvantes en la disparidad entre lo planeado y lo ejecutado.

Palabras clave: Transparencia, Implementación de políticas públicas, Corrupción, Diseño institucional, México. 


\title{
WHAT HAS GONE WRONG?: \\ FINDINGS ON THE IMPLEMENTATION OF PUBLIC POLICIES FOR TRANSPARENCY STARTING FROM AN INSTRUMENTAL CASE
}

\begin{abstract}
What happens once a public decision is put into practice? The answer to this question lies in the field of public policy implementation, an area studied in a discontinuous manner over time. After a theoretical account, this article explores - starting from an instrumental case set in a Mexican subnational government which has implied bringing a legislative decision related to transparency to reality. Among the findings it is prominent situations of a political nature, agreements between groups and concentration of power in few actors, for example, as contributing elements to the gap between what was planned and executed.
\end{abstract}

Keywords: Transparency, Public policy implementation, Corruption, Institutional design, Mexico. 


\section{INTRODUCCIÓN}

El presente es un estudio sobre implementación de políticas públicas, tema relevante del que poco se ocupa la investigación nacional y, en general, la latinoamericana. Decía Elmore que "en la mayoría de los casos es imposible afirmar si las políticas fracasan porque se basan en ideas erróneas, o si fracasan porque son buenas ideas mal llevadas a la práctica" (Elmore 2000: 186). En políticas públicas, la diferencia entre lo planeado y lo ejecutado se conoce como brechas de implementación. Éste es, de manera muy aproximada, el problema al que nos enfrentamos.

Realizar un estudio sobre implementación de políticas públicas conlleva varias complicaciones: la ausencia de un marco teórico unificado y la histórica discrepancia entre las unidades de análisis empleadas en los estudios sobre administración pública y los de implementación de política pública. A esto habrá que sumarle que el cuerpo de estudios parcamente ofrecen casos a partir de la realidad latinoamericana: aprendemos de política pública siguiendo las traducciones y conociendo los casos estadounidenses o europeos cuyo contexto dista al propio, en esta parte del continente.

El presente artículo se desarrolla a partir de la siguiente pregunta detonante: ¿Qué implica llevar a la práctica una decisión pública (en este caso, de naturaleza legislativa)? Para ello, en la primera parte se abordan someramente los marcos teoréticos existentes sobre la implementación de políticas públicas. Posterior a esto, el lector encontrará el recuento de los hechos principales alrededor del episodio de la crisis que enfrentó el gobierno subnacional de Coahuila, entidad federativa ubicada en el norte de México que, contando con la segunda mejor Ley de Acceso a la Información Pública en el país (CIDE 2010) paradójicamente, se presentó el quiebre financiero producto de la falta de transparencia en el manejo de las finanzas públicas. Es, pues, un caso donde el diseño organizacional y el entramado institucional se ponen a prueba en un contexto de crisis. En la parte final, se destacan los principales hallazgos de este recorrido.

\section{LA IMPLEMENTACIÓN COMO PROBLEMA DE POLÍTICA PÚBLICA}

Implementar es, desde el plano empírico, poner en funcionamiento, aplicar métodos o medidas para llevar a cabo algo. En el campo de la política pública se trata de un fenómeno complejo y, hasta hace relativamente poco tiempo, sin análisis. Esto último ha provocado un conocimiento disperso y poco consistente. 
En el texto seminal del tema que nos ocupa, para explicar la complejidad de la implementación, Pressman y Wildavsky (1998) reiteradamente incluyeron ilustraciones de Rube Goldberg, satirizando lo que sucede entre la decisión de que algo debía hacerse y la entrega del bien o la prestación del servicio público. Cuarenta años después, las complicadas y elaboradas estructuras del ilustrador Rube Goldberg que desarrollan una simple operación, pudieran mantener su vigencia.

Sin embargo, que en la práctica la implementación sea algo complejo, dista de la comicidad que puede encontrarse en el trabajo el ilustrador: la disparidad entre lo planeado y finalmente implementado, implica una gestión pública injusta que no podría, de forma alguna, generar valor en los bienes o servicios que provee. Por esto, comprender la implementación de las políticas públicas importa más allá del campo académico o el plano teórico.

Al respecto, Marilee S. Grindle llama brecha en la implementación a "la disparidad qe frecuentemente hay entre lo que se enuncia como política y lo que en realidad se ejecuta" (Grindle 2009: 33), y califica como sensato todo esfuerzo que pretenda hacer más implementable una política pública.

\section{ESTUDIOS SOBRE IMPLEMENTACIÓN DE POLÍTICAS PÚBLICAS}

De acuerdo con Paul Berman,

[...] el análisis de la implementación es el estudio de por qué las decisiones provenientes de la autoridad (sean éstas políticas, planes, leyes u otras) no conducen necesariamente al logro de los resultados previstos. Para plantearlo en términos más positivos, el análisis de la implementación es el estudio de las condiciones bajo las cuales las decisiones de la autoridad conducen efectivamente a los resultados deseados (Berman 2000: 286).

Antes, debido a que el enfoque predominante de las políticas públicas se concentraba en el problema: "el análisis del proceso de las políticas tendía a preocuparse por temas como cuán racional, abierta o justa era o podía ser la toma de decisiones" (Parsons 2007: 477). Qué hacer después de tomada la decisión, fue algo hasta entonces ignorado. Posterior a ese momento, la década de los setenta, el conocimiento al respecto ha presentado diferentes estadios y ha avanzado a velocidades diferentes: hay un crecimiento cuantitativo y cualitativo- de los estudios sobre implementación desde los años setenta y hasta entrados los años ochenta; después, en la década de los noventa y principios de este siglo, la productividad académica y los avances 
teoréticos al respecto, fueron escasos quizás, como dice Susan M. Barret (2004, referida por Bastien 2009: 666), por la creencia de que la reformas provenientes de la Nueva Gestión Pública (NGP) habrían de solucionar las fallas en la implementación.

\section{DE LAS COMPLEJIDADES INHERENTES AL ESTUDIO DE LA IMPLEMENTACIÓN}

La literatura sobre implementación identifica como complejo su estudio por las siguientes tres razones: 1) su conceptualización; 2) la unidades de análisis empleadas; 3) los pocos casos/muchas variables que pueden estudiarse.

Respecto a la construcción del concepto, no existe uniformidad sobre qué debe entenderse por implementación: hay tantas definiciones como autores han estudiado el tema. Dada su característica polisémica cabría, al menos, "un doble sentido de implementación: es el proceso de convertir un mero enunciado mental (legislación, plan o programa de gobierno) en un curso de acción efectivo y es el proceso de covertir algo que es sólo un deseo, un efecto probable, en una realidad efectiva" (Aguilar Villanueva 2000: 47).

El señalamiento de la ausencia de una integración conceptual de los estudios sobre la implementación pública no es nuevo. Ya en su texto de 1981, La Implementación de la Política Pública: Un Marco de Análisis, Paul A. Sabatier y Daniel A. Mazmanian señalaban algunos esfuerzos realizados buscándola: los estudios de Rein y Rabinovitz, los de Bardach, los de Berman y, finalmente, los de Van Meter y Van Horn (Sabatier y Mazmanian (2000 325-327), como ejemplos más destacados.

Para Sabatier y Mazmanian, en su propuesta de marco conceptual del proceso de implementación,

[...] la función central de análisis de la implementación consiste en identificar los factores que condicionan el logro de los objetivos normativos a lo largo de todo el proceso. Estos factores pueden dividirse en tres categorías amplias: 1) la tratabilidad del o de los problemas a los que se dirige el estatuto ${ }^{1}$; 2) la capacidad del estatuto para estructurar apropiadamente el proceso de implementación; y 3) el efecto neto de las diversas variables políticas en el apoyo a los objetos estatutarios (Sabatier y Mazmanian 2000: 329).

Diversos análisis existentes sobre implementación lo ubican como la

1 Para Sabatier y Mazmanian, la implementación es el cumplimiento de una decisión política que, por lo general, se plasma en un estatuto. De aquí el empleo de este vocablo. 
consecuencia de tres etapas (hacer la política, implementar la política, resultados), aun cuando la evidencia muestra que la relación de etapas dentro del proceso es más complejo que esto (Berg y Colton 1985). Hay quien señala que, incluso, el propio análisis por etapas, muy socorrido en el estudio de políticas públicas no ofrece claridad al respecto: si "la mayor parte de los actos administrativos o quizás todos ellos hacen política y cambian la política al intentar implementarla [...] [entonces debiéramos] analizar la implementación como parte del policy-making" dice Lindblom (citado por Aguilar Villanueva 2000: 34).

La carencia de un cuerpo teórico coherente relacionado con la implementación de políticas públicas también ha sido señalado por Berman (2000).

En relación con las unidades de análisis, Hasenfeld y Brock (1991), en su esfuerzo por unificar la producción literararia sobre implementación, advierten la utilización de, al menos, cuatro diferentes unidades de análisis, lo que dificulta su sistematización. Por una parte, se encuentran los estudios cuya unidad de análisis engloba incentivos, la autoridad legal y el diseño técnico de los instrumentos empleados en la política; un segundo grupo de estudios, emplean como unidad de análisis el trabajo en red interorganizacional, ya sea vertical u horizontal; un tercer grupo se enfoca en los procesos intraorgánicos. Finalmente, una cuarta clase de estudios emplean como unidad de análisis a los actores individuales.

Identificado por Lijphart (citado por Goggin 1986), el problema "muchas variables/pocos casos" dificulta el diseño de investigaciones cuantitativas y, con ello, el desarrollo del conocimiento sobre implementación de políticas públicas. Bajo esta denominación, se pretende retratar la situación a la que se enfrenta el investigador que encuentra, al mismo tiempo, pocos casos de estudios comparables entre sí (cada experiencia es única) y muchas variables, a las que pudiera deberse la falla o éxito de la propia experiencia, de cada caso.

Para el caso particular, aplicable solo en estudios cuantitativos, se han propuesto (Homans y Lijphart, citados por Goggin 1986) tres estrategias: decrecer el número de variables incluyendo únicamente las críticas ${ }^{2}$, incrementar el número de casos e introducir elementos de control.

2 Goggin (1986: 332), propone como variables críticas independientes: 1) el contenido de la política pública; y 2) el setting, es decir, la gente y organizaciones involucradas en la implementación; y como variables críticas dependientes: 1) duración; y 2) cambio. 


\section{Clasificación POR generaciones Del CONOCIMIENTo EXISTENTE}

Los estudios elaborados en poco más de cuatro décadas, en los cuales se han presentado momentos de estancamiento y momentos en los que el interés por el tema revive (Cairney 2009), han sido clasificados por diversos autores para facilitar su comprensión.

Para Milbrey McLaughlin (1987), por ejemplo, son dos las generaciones que deben considerarse: la primera, donde se descubre que la implementación es un problema, y la segunda, donde el trabajo de campo comienza a derivar interesantes contribuciones. Para ese entonces, el texto se publica en 1987, McLaughlin preveé existirá una tercera generación de estudios enfocados en los problemas de microimplementación y de macroimplementación ${ }^{3}$. En 1991, Hasenfeld y Brock propusieron tres categorías: el trabajo que podía agruparse en las teorías top-down, las teorías de bottom-up y las interativas. A partir de entonces, las categorias top-down y bottom-up son aceptadas y reproducidas de manera generalizada.

De acuerdo con el estudio introductorio sobre la implementación elaborado por Luis F. Aguilar Villanueva (1993, 1996, 2000), pueden identificarse tres generaciones de estudios: la primera, producto de la frustración provocada en académicos y practicantes por la nula incidencia de las políticas públicas en los objetivos propuestos; la segunda, que enfatizó la idea de una implementación desde abajo como una reacción al diseño e implementación de las políticas públicas bottom-up donde, según se consideró, se encontraba el problema; la tercera, la implementación desde arriba o top-down.

Para las políticas públicas, al menos en Estados Unidos, la década de los años sesenta fueron de optimismo: se generaron legislaciones para erradicar racismo, pobreza y desigualdad. Sin embargo, dicho optimismo duró poco: para la siguiente década, quedaba demostrado que aquellas "suposiciones de que las leyes se implementaban por ellas mismas, ya no parecían válidas [...] [por lo que] la implementación de las políticas públicas comenzaron a atraer la atención” ${ }^{4}$ (Sarbaugh-Thompson y Zald 1995: 25).

De esta generación, "uno de los trabajos más citados [...] es el de Martha Derthick (1972), New Towns In-Town [...] [por haber] lanzado por primera vez la pregunta teórica Why a federal program failed?, por qué fallan los programas federales" (Aguilar Villanueva 2000: 37). Pressman y Wildavsky, por cierto, la reconocen en el prefacio de su estudio. Derthick

3 Ambas, la macroimplementación y la microimplementación, son estudiadas por Berman, y aquí están descritas a partir de Aguilar Villanueva (2000).

4 Traducción del autor. 
estudia un programa iniciado en 1967, durante la presidencia de Johnson, por el que se construirían comunidades modelo en terrenos sobrantes de la propiedad federal en Washington, San Antonio, Atlanta, Michigan y San Francisco. El estudio refleja una situación donde "hay un aparente acuerdo al principio, y luego, muy pronto, surgen desavenencias que bloquean el programa” (Pressman y Wildavsky 1998: 175).

Conforme avanzaba la década de los setenta, se comprendió que "el problema central se localizaba en la implementación de las políticas públicas más que en su diseño" (Aguilar Villanueva 2000: 25) que los implementadores tenía una mayor participación y que, incluso, la sola obediencia no garantizaba el éxito de lo planeado. Comienza, entonces, la identificación de factores tales como la complejidad de la política, el tamaño de la organización implementadora, el compromiso existente en ellas, es decir, las principales aportaciones de esta generación. Cómo resolver los problemas de implementación es un tema poco explorado en esta generación: comienza a intuirse, sin que exista un buen desarrollo, que los problemas podrían resolverse "clarificando la relación entre metas y medios" (Comfort 1981:C77), definiendo no sólo el qué, sino el cómo. Otra característica de esta etapa, como apunta Goggin (1986), es que la mayor parte de los estudios se ocupan de una sola autoridad responsable de la implementación de una política pública, ya sea en una o en varias localidades. No fue, pues, una generación optimista "ni tenía razón para serlo, aunque su presunción inicial de las políticas públicas como 'zona de desastre' los condujo a generalizaciones teóricamente discutibles" (Aguilar Villanueva 2000: 32). Esto se debe a que el concepto de fracaso o fallo de una política pública, en la óptica contemporánea al menos, es criticado toda vez que es dificil tomar decisiones acerca de su efectividad sin una implementación total de la política pública (Corbett y Lennon, citados por Cooley 2010: 68).

La segunda generación, dedicada a realizar estudios en una lógica topdown, se caracteriza por enfocarse en cómo el proceso de implementación puede estructurarse para conseguir los objetivos (Hasenfeld y Brock 1991: 452). Para este momento, es evidente que "los fracasos [el no alcanzar los objetivos] pueden deberse a una defectuosa implementación" (Aguilar Villanueva 2000: 32).

El estudio emblemático de esta generación, con el que se considera inicia formalmente el estudio de la implementación, fue el elaborado por Pressman y Wildavsky en 1973. El trabajo de estos dos autores es producto de "entrevistas con los actores pertinentes y [el] análisis de documentos de políticas públicas y otros temas relacionados durante tres años con el objetivo de determinar dónde se cometieron errores" (Parsons 2007: 484). 
La experiencia descrita es la de la agencia Administración del Desarrollo Económico (ADE), creada por el Congreso de los Estados Unidos a mediados de la década de los sesenta ${ }^{5}$, responsable de implementar un programa generador de empleo a través de la provisión de obra pública. Para experimentar la implementación se eligió la ciudad de Oakland.

La primera gran conclusión a la que se llega en el estudio en cita resume la reconstrucción que se hace, en los primeros cuatro capítulos, de la experiencia de la ADE: "Cuanto más larga sea la cadena de causas, más numerosas serán las relaciones recíprocas que se establezcan entre los eslabones y más compleja se volverá la implementación" (Pressman y Wildavsky 1998: 56). Se presentan, además, dos términos acuñados en el campo de la implementación de la política pública:

Denominamos punto de decisión a cada ocasión en que tenga que registrarse un convenio para que el programa continúe. Y le damos el nombre de certificación a cada caso en el cual se requiera que un participante exprese su consentimiento por separado. La suma del número de certificaciones necesarias que se contienen en los puntos de decisión durante la vigencia del programa, da al lector una idea de la tarea que se emprende para lograr la implementación. (Pressman y Wildavsky 1998: 57).

Por vez primera hay un reconocimiento expreso de que la mera descripción del fracaso poco provee en la solución del problema. Habría que penetrar más profundo en las raíces del fracaso, pues "hay sorpresa y, por tanto, impulso a la búsqueda de explicación, cuando se malogra una política que desde su comienzo gozó de consenso serio, disponibilidad de recursos, criterios legislativos precisos y compatibles y la oposición fue inexistente" (Pressman y Wildawsky, citados por Aguilar Villanueva 2000: 43).

A partir de este trabajo, la implementación deja de ser concebida como una consecuencia lógica y necesaria al diseño de la política pública: "lo que esperamos demostrar es que lo aparentemente sencillo y directo es realmente complicado y retorcido" (Pressman y Wildavsky 1998: 178); y, como problema, deja de ser considerada como la excepeción, para volverse la regla: "alguien que vaya siempre en busca de circunstancias insólitas y acontecimientos dramáticos no puede apreciar lo dificil que es hacer que acontezca lo ordinario" (Pressman y Wildavsky 1998: 53). Si bien se reconoce

5 Se genera a través de la Ley de Desarrollo Económico y Obras Públicas, aprobada el 26 de agosto de 1965. 
que "pocos programas podrían emprenderse si se tuvieran que especificar de antemano todos los participantes [...] [por lo que] [...] algo tiene que dejarse al avance de los acontecimientos" (Pressman y Wildavsky 1998: 176), se advierten ya características que provocaron una implementación deficiente. Primero, la multiplicidad de participantes y perspectivas. Esto se debe a una de las siguientes razones: 1) la incompatibilidad directa con otros compromisos, es decir, los participantes podrían estar de acuerdo en los méritos de la política a implementar pero descubrir que es incompatible con otras metas de la organización; 2) no hay incompatibilidad directa, pero sí una preferencia por otros programas; 3) existen compromisos simultáneos con otros proyectos; 4) existe subordinación a otras entidades que no ven la urgencia de la implementación de la política pública; 5) existen diferencias de opinión sobre la jefatura y funciones propias de la organización; 6) existen diferencias legales y de procedimiento entre las agencias participantes; 7) carecer de recursos para apoyarlas o, en términos de los autores, existe acuerdo junto con la falta de poder (Pressman y Wildavsky 1998: 184-189). Segundo, la multiplicidad de decisiones que disminuye la probabilidad de éxito de la política pública. Tercero, la existencia de más de una meta y más de un curso de decisiones. Cuarto, el surgimiento de decisiones inesperadas. Finalmente, quinto, la demora que toma la implementación del programa que los autores relacionan con los recursos a emplearse.

El texto identifica "dos remedios administrativos muy socorridos" (Pressman y Wildavsky 1998: 220) que en verdad son paliativos a los problemas de implementación: 1) salir de la burocracia y 2) la coordinación entre participantes.

Salir de la burocracia significaría: establecer una nueva organización, lo que significa contratar nueva gente, elaborar reglas nuevas y elaborar modelos de operación que se considere eliminan los problemas ya identificados. Significa, también: a) un trato diferenciado por tiempo limitado; b) gastos adicionales; c) la inexperiencia de la organización creada para afrontar el problema. Buscar la libertad/independencia de la organización significa dos costos: a) por lo general, una estructura plural de dirección ejecutiva, lo que implica una franca invitación a que una diversidad de partes interesadas exijan tener representación; y b) se pierde el contacto con las fuerzas políticas necesarias para mantener el empuje de la organización (Pressman y Wildavsky 1998: 221-222).

Coordinación entre participantes, dicen los autores, resulta una palabra de engañoza sencillez. Y agregan:

[...] cuando un burócrata le ordena a otro que coordine un plan de 
acción, quiere decir que éste debe ser aclarado con otros participantes oficiales que tienen cierto interés en el asunto, lo cual entraña una manera de compartir la culpa en el caso de que las cosas salgan mal [...] es también una forma de aumentar la capacidad de pronosticar el aseguramiento de cada acuerdo necesario para acometer una acción futura [...] Coordinación significa conseguir lo que no tenemos. Significa crear unidad en una ciudad que está desunida. (Pressman y Wildavsky 1998: 227-228).

Finalmente, se identifican dos clases de medidas que pueden tomarse: 1) considerar medios más directos para alcanzar los fines; 2) centrar la atención, tanto en la creación de la maquinaria de la organización para ejecutar el programa como en su lanzamiento (Pressman y Wildavsky 1998: 240).

Por su parte, para Rein y Rabinovitz "los encargados de la implementación deben tomar en cuenta el resultado del proceso [legislativo] y asumir que uno de los imperativos formales de los funcionarios públicos será obedecer la ley tal y como haya sido formulada” (Rein y Rabinovitz 2000: 150).

A lo anterior, los autores le denominan el imperativo legal. Este va aparejado con el imperativo racional-burocrático, integrado por la concepción, de parte de los burócratas, de: 1) la viabilidad de la política pública; y 2) de la coherencia que detecte con los principios que rigen su actividad. Hay, en el trabajo de Rein y Rabinovitz, un tercer imperativo denominado imperativo consensual, que incluye la cooperación entre actores involucrados en la elaboración de la política pública (Rein y Rabinovitz (2000: 153-159). Desde la perspectiva de estos autores, una decisión correcta al inicio de la elaboración de la política pública es lo que garantizaría el éxito. Elaboran, incluso, una lista de factores necesarios para este fin: 1) la fuerza y el prestigio del comité legislativo en el que el proyecto de ley se origina; 2) la experiencia de los miembros del Comité generador de la ley de la que se desprende la política pública; 3) el grado en que los desacuerdos existentes durante el proceso legislativo fueron impugnados; 4) el nivel de apoyo a la ley de parte de los legisladores y las comunidades donde se implementaría el resultado de la ley (Rein y Rabinovitz (2000: 151).

Derivado de los estudios contenidos en esta generación, de acuerdo con Cairney (2009: 357), el éxito de una política pública requiere seis condiciones: 1) que exista un entendimiento de los objetivos de la política y se le identifican como consistentes; 2) que la política funciona como se espera al momento de implementarla; 3) que las tareas por hacer son específicas y claramente comunicadas; 4) que los recusos (inlcuida la voluntad política) 
están comprometidos; 5) que se mantenga el apoyo de los grupos de interés; y 6) que los factores externos al proceso (situaciones socioeconómicas, por ejemplo) no socavan significativamente la implementación.

La generación tercera generación, enfocada a los estudios bottom-up, se caracteriza por estudiar la implementación desde la perspectiva de las organizaciones y los actores responsables de llevar a la práctica la política pública (Hasenfeld y Brock 1991: 452). Hasta el trabajo de Myrtle (1983), dentro de esta generación, la mejora en la implementación también podría lograrse con medidas relacionadas: administración por objetivos, presupuestación base cero, plan de pagos basados en desempeño. Es precisamente Myrtle quien cambia esta perspectiva al proponer un marco que considere la implementación como un problema social y de cambio organizacional y, con esto, la macroimplementación y la microimplementación. La primera de ellas, la macroimplementación, se enfoca en la necesaria colaboración entre organizaciones; la segunda, en términos de McLaughlin, se presenta donde "en el proceso de implementación, el cambio es, ultimadamente, el problema de la unidad más pequeńa. A cada paso del proceso, una política es transformada de acuerdo cómo los individuos la interpretan y responden a ella (traducción del autor)" (McLaughlin 1987: 174), es decir, la personas encargadas de la implementación.

A partir de la segunda generación, existe una visión más ecléctica del problema de la implementación al reconocérsele su naturaleza variable y la presión existente entre las dimensiones política y administrativa o de gestión (Goggin 1986: 328).

Toda vez que "los modelos de abajo hacia arriba enfatizan bastante el hecho de que los implementadores con trato directo con el público aplican las políticas con discrecionalidad" (Parsons 2007: 489), el análisis de la discrecionalidad comienza a ocupar un lugar importante en los estudios sobre el tema. Al respecto, Elmore, criticando la propuesta dominante de la generación top-down, señala:

[...] cuando la implementación consiste esencialmente en el control de la discrecionalidad, tiene como consecuencia, por un lado, que se reduzca la confianza en el despliegue de los conocimiento y habilidades en el nivel operativo y, por otro, que se dependa más de soluciones abstractas y homogéneas (Elmore 2000: 267).

Entonces, para los estudios que pueden incluirse en esta generación, tal y como se refiere en los trabajos de Lipsky, "el control sobre la gente no es la vía para la implementación efectiva” (Parsons 2007: 488). En otras 
palabras, habría que dejarlos tomar decisiones con base en lo que perciben al momento de implementar la política pública.

Sobre la importancia de la organización, Hepburn y Goodstein (1986) identifican como impedimentos de una implementación exitosa: la resistencia al cambio del personal clave cuando éste siente que la tarea va en contra de sus creencias y la generación de riesgo a su situación de poder y autoridad dentro de la organización.

\section{¿QUÉ SALIÓ MAL? COAHUILA, MÉXICO: DE LA MEJOR LEY EN TRANSPARENCIA AL VIRTUAL QUIEBRE FINANCIERO POR MANEJO OPACO DEL DINERO PÚBLICO}

Para comprender lo relacionado con la transparencia y el acceso a la información pública en México deberá tenerse en cuenta la naturaleza federalista del país: sin contradecir un pacto nacional consagrado en la Constitución, cada orden subnacional (llamado entidad federativa o estado; 32 en total) tiene un gobierno integrado por un órgano Legislativo, uno Ejecutivo y uno Judicial. Aun cuando por razones financieras y presupuestales pudiera pensarse que los gobiernos subnacionales están subordinados al nacional, esto no es así: cada entidad federativa disfruta cierto grado de autodeterminación y autonomía en algunas materias. La transparencia y el acceso a la información, entre ellas. Esta situación ha generado que, a lo largo de poco más de diez años, en México existan más de una treintena de experiencias a manera de laboratorios para el observador de políticas públicas.

El caso aquí referido se ubica en el gobierno subnacional del estado de Coahuila. El titular del Ejecutivo, el Gobernador como se le denomina, no terminó su periodo de seis años, pues fue designado por el Partido Revolucionario Institucional (PRI) como su líder nacional. $\mathrm{Su}$ posicionamiento en el contexto político, no es menor: se trataba de la posición formal más cercana al entonces contendiente con mayores posibilidades de ser Presidente de la República, Enrique Peña Nieto.

El 2 de diciembre de 2011, en su carácter de Presidente del Comité Ejecutivo Nacional del PRI, Humberto Moreira Valdés (para entonces Gobernador con licencia de Coahuila) dirigió un mensaje corto a quienes asistían a la XXVIII Asamblea Extraordinaria del Consejo Político. Renunciaba a esa posición nueve meses después de haberla asumido:

Hoy llego con ustedes para decirles que presento mi renuncia como Presidente del Comité Ejecutivo Nacional del Partido. La presento [...] porque no voy a permitir que por una guerra mediática se esté 
tratando de dañar a mi partido, que tantas oportunidades me ha dado $[\ldots]$.

La guerra mediática aludida por Humberto Moreira Valdés detona menos de una semana antes de las elecciones para elegir Gobernador de Coahuila, el 3 de julio de 2011, como consecuencia de la cobertura hecha por medios de comunicación sobre las denuncias penales presentadas por integrantes del Partido Acción Nacional (PAN). Posterior a esas denuncias, y en esto radica el seguimiento dado por los medios de comunicación, se daría a conocer: 1) un endeudamiento superior a lo permitido por las normas aplicables, mismo que hasta entonces había sido negado y ocultado; 2) la falsificación de documentos para la tramitación de una parte del monto total de la deuda; y 3) lo que sería una red de corrupción que involucra servidores públicos del Estado de Coahuila y la Secretaría de Hacienda y Cuenta Pública (SHCP) del Gobierno Federal. Como consecuencia del endeudamiento excesivo, el Congreso del Estado, con mayoría del PRI: 1) reformó algunas leyes dotándolas con efectos retroactivos, intentando enmendar toda acción realizada contraria a la ley; y 2) aprobó, sin que existiera información sobre la aplicación del recurso debido, un decreto para el refinanciamiento de la deuda. Estos dos movimientos, por cierto, han sido señalados por la oposición como ilegales ante tribunales, estando pendiente la resolución jurisdiccional.

Guerra mediática o natural cobertura de una serie de hechos con interés social y, por ende, noticioso, el problema de la deuda en Coahuila detonó un año antes de las elecciones presidenciales del 2012. Humberto Moreira Valdés, aun cuando no se ha acreditado su participación directa, tenía pocos meses de haber asumido la presidencia del partido que, de acuerdo con diversas encuestas, tiene las mayores oportunidades de ganar las elecciones. No es asunto menor.

El episodio es significativo, al menos, desde tres aproximaciones: primera, en lo político nacional, es la separación anticipada del líder formal del partido político con las más altas probabilidades de obtener la Presidencia de la República; segunda, en lo político estatal, incide en el liderazgo multidimensional depositado en quien fuese el Gobernador de Coahuila. Hay una tercera aproximación: la relacionada con la gestión de lo público.

Las primeras dos aproximaciones son de aprovechamiento para la disidencia en la arena política. Este trabajo pretende, apartándose de ellas, optar por el tercero de los caminos cuyas lecciones y conclusiones tendrán un más amplio espectro de beneficiarios. 
Posterior al caso Coahuila, en la agenda pública se coloca el tema de los mecanismos para la adquisición de la deuda. El tema de la transparencia, ya en la agenda nacional desde al menos diez ańos antes, regresa como prioridad. En el caso particular de Coahuila, comprender las implicaciones del monto reconocido de la deuda contraída, alrededor de US \$3.000.000.000, a febrero de 2012, es apenas el inicio: 1) a lo largo de cinco años se negó su existencia; 2) no hubo, producto de alguno de los mecanismos de acceso a la información, indicio alguno que pudiese advertir qué estaba por suceder; 3) como ya quedó asentado, una parte se tramitó presentando documentos apócrifos y, de la deuda en general, no es claro el destino del monto total; 4) la Ley de Deuda se reformó en repetidas ocasiones para aplicarla de manera retroactiva buscando la convalidación de actos ya consumados; y 5) el Congreso del Estado aprobó lo necesario para el refinanciamiento de la deuda sin tener a la vista documentación que soportara el origen y el destino de los recursos. En todo esto, el denominado común fue la falta de transparencia.

Todo lo anteriormente descrito se presenta en un estado donde existen los mismos o más controles legales y orgánicos para el control del gasto. Detona en un Estado donde al Instituto Coahuilense de Acceso a la Información Pública (ICAI), órgano constitucional autónomo autoridad en la materia, se le reconocen seis clases de autonomías. Detona en el Estado cuya política pública de transparencia surge de la segunda mejor ley de acceso a la información pública del país ${ }^{6}$. Entonces ¿qué sucedió en Coahuila?

\section{Primer episodio de la transparencia en Coahuila}

Nelly Herrera, activista social en Coahuila por más de 30 años, recuerda los orígenes de lo que sería la transparencia en Coahuila: "Allá en el 2001, por cuestiones políticas, de ascenso al poder, en este caso del Gobernador Enrique Martínez y Martínez, antecesor de Humberto Moreira Valdés, trató de crearse una imagen democrática y hace reformas de fondo tanto electorales como de participación ciudadana [...] [de donde se derivaría la Ley de Acceso a la Información Pública]" (Herrera, en entrevista). Además de la participación ciudadana, en Coahuila, la transparencia se origina con la firma de un convenio interinstitucional suscrito por los tres poderes del Estado (el Ejecutivo, el Legislativo y el Judicial) y el Instituto Electoral ${ }^{7}$.

6 En el estudio Métrica de la Transparencia 2010 (CIDE 2010), se evaluaron cuatro aspectos de todos los organismos de acceso a la información: normatividad, portales, usuario simulado y capacidades institucionales. De acuerdo con los resultados de este estudio, Coahuila tiene la segunda mejor ley. En portales, obtuvo el lugar 23. En usuario simulado, el lugar 13. En capacidades institucionales, el lugar 22. Más información puede consultarse en www.metricadetransparencia.cide.edu

7 No se tiene conocimiento de un ejercicio participativo como éste en alguna otra 
En noviembre de 2004, se constituye el Órgano Superior de la organización que promovería y vigilaría la transparencia: la primera generación de consejeros se integró por Eloy Dewey Castilla, Alfonso Villarreal Barrera y José Manuel Gil Navarro.

Del diseńo legislativo original del organismo para la transparencia en Coahuila, tres datos fundamentales: primero, el encargo de Consejero duraría siete años con excepción de la primera generación, en la que existió diferenciación en los periodos de manera escalonada para evitar la renovación total cada periodo; segundo, el encargo de Presidente, decidido entre los tres, duraría lo que la consejería de quien la ostentara; tercero, no existía regulación sobre si un consejero podía o no ser ratificado en su puesto.

La primera decisión que tomaron los tres consejeros fue la elección del Presidente, con su voto y el de Manuel Gil, sería Eloy Dewey. La unanimidad no se logró por el voto disidente de Alfonso Villarreal. Las diferencias que pudieran existir entre los Consejeros no superaban sus coincidencias. Dewey percibía en el Consejo una ferviente convicción en el tema. "Éramos idealistas o ingenuos: pero lo éramos. Creíamos en un tema, que era para bien de una democracia y de una sociedad más participativa. Mis compañeros en un principio lo tenían, y eran congruentes con lo que yo pensaba" (Dewey, en entrevista).

Pero la duración de la presidencia, que de acuerdo con la Ley coincidiría con la duración de la consejería de quien la ostentara, dificultaba la situación:

Justo antes de asumir la consejería, tuvimos una reunión [...] los tres consejeros y comenzamos a analizar la conveniencia de que la presidencia fuera de dos años y de carácter rotativo, por el desgaste en el tema que representaba para el propio Presidente y que consideramos que un elemento esencial del sistema democrático era la posibilidad de que todos pudiéramos ser Presidente. Y, en aquel entonces, se tomó un acuerdo: que íbamos a tener una presidencia rotativa, dos, dos y dos años [...] el desconocimiento de ese acuerdo [de parte de Eloy Dewey] fue uno de los factores determinantes, para que la relación fuera contaminándose (Villarreal, en entrevista).

Todos querían ser Presidente y hubo un acuerdo verbal contrario a lo establecido por la Ley aunque se argumenta por parte de los involucrados que no fue para quebrantarla. Se había plantado la semilla que germinaría

entidad. Da muestra del acuerdo político entre los principales actores dentro de una entidad federativa mexicana. 
a los tres años, con la salida anticipada de Eloy Dewey.

En esta primera etapa, el énfasis estuvo en la selección de personal: se buscó personal con un perfil adecuado bajo criterios técnicos a pesar de que pasaban siempre por un filtro político, recuerda Cárdenas (en entrevista). Ese filtro político se encontraba dentro del ICAI, en el Consejo General. Las decisiones sobre el ingreso de personal, la estructura y las funciones no estaban alejadas de una lógica política. En este panorama, el ICAI trabajó sin mayores complicaciones. Desarrolló e implementó una fuerte campaña de publicidad y propaganda invitando a la comunidad a ejercer el derecho de acceso a la información, se construyó la relación con los sujetos obligados, se mantuvo la relación con medios de comunicación y sociedad civil organizada.

Fue un inicio tan complicado como cualquiera, sin mayores sobresaltos salvo los propios del trabajo:

[...] [después del primer año se fue avanzando] a pasos lentos, un poquito con el lastre de la burocracia de las instancias públicas [...] fue avanzando desde el punto de vista organizacional, desde el punto de vista estructural, independientemente de las fuerzas internas que se movían desde lo político y desde el perfil de cada uno de los tres líderes de la organización, que eran los tres consejeros. Y otro líder satelital, que en este caso era la Secretaría Técnica (Cárdenas, en entrevista).

Quien entonces fue Director General del ICAI, al respecto dice:

Creo que lograron avanzar, hasta que vino la intención de sacar al presidente [Eloy] Dewey. La intención, que no sabemos de dónde vino, que él denunció y todo. Ese sisma, fue el que comenzó a romper con el avance que se tenía (en entrevista).

El segundo año de existencia del ICAI coincide con el primero de la administración de Humberto Moreira Valdés. En palabras de Dewey, entonces Presidente del ICAI,

En 2005, funcionó bien [el ICAI], 2006 funcionó bien. Hasta que llegó el segundo año [del Gobierno de Humberto Moreira Valdés] donde comenzaron los problemas de carácter institucional, en la cúpula del Consejo [General del ICAI] por los intereses propios. Al haber creado ya una Institución, una estructura funcional, tan funcional que es fecha que opera (en entrevista).

La relación entre los tres consejeros cabeza del ICAI fue cambiando, 
siendo Eloy Dewey quien se llevó la peor parte:

Comencé a percibir, en los compañeros, un alejamiento del tema; un restarle compromiso auténtico. ¿Cómo se reflejaba? En hacer reuniones alejadas de la capital, ocultar el tema, tener un bajo perfil [...] inclusive, a mí me tuvieron un vocero, o comenzar a ver la posibilidad de una vocería [...] comenzamos a recibir solicitudes sobre mi persona y sobre la Institución, a ver si encontraban por ahí algún lado vulnerable [...] mi celular lo cancelan, sin decir 'agua va' [para restringir la comunicación] [...] son formas de intimidación (Dewey, en entrevista).

Las diferencias entre los Consejeros comenzaron a afectar el desarrollo del trabajo de la organización pública:

Yo creo [recuerda Cárdenas, entonces Director General] que el instituto se enfrascó en una lucha política muy fuerte, con mucho peso mediático, pero a la vez tratando de arrancar una estructura administrativa [...] se enfrascó en un, diría yo, un galimatías, en un laberinto. No estábamos nadie preparados a ese nivel de exposición mediática, ni a ese nivel de presión política $y$, entonces, lo administrativo pasaba a segundo plano (Cárdenas, en entrevista).

Pero las diferencias parecían irreconciliables. Alfonso Villarreal recuerda,

Tuvimos la madurez y tuvimos la entereza de reconocer que no nos poníamos de acuerdo y que necesitábamos de alguien que nos pusiera de acuerdo [...] [en aquél momento] tenemos puntos de incompatibilidad, pero llegamos a un punto compatible: jestamos de acuerdo que no nos ponemos de acuerdo?; ¡estamos de acuerdo que no nos vamos a poner de acuerdo?; ¡estamos de acuerdo que tenemos que ponernos de acuerdo? (en entrevista).

Se contrató un servicio externo de asesoría para disminuir las diferencias entre los Consejeros.

Estaban los tres consejeros recibiendo ayuda externa a través de la consultoría cuando se vuelve evidente que las diferencias con el entonces Presidente del ICAI habían superado al propio instituto: el Congreso del Estado entra en la disputa.

En noviembre de 2006 se presenta una iniciativa de reforma a la Ley del ICAI por la que se pretende acortar a dos años la duración de la presidencia y hacerla de carácter rotativo entre quienes eran consejeros. 
Aun cuando se considere que el cambio fue impulsado desde fuera, requirió de la aceptación de quienes ya estaban dentro, es decir, los dos consejeros: Gil y Villarreal. Dewey, aun Consejero Presidente, comienza una lucha solitaria para defender su presidencia. Aprobada la reforma, los consejeros Manuel Gil Navarro y Alfonso Villarreal Barrera, la pusieron en práctica en febrero de 2007 cuando, por votación de dos contra uno, remueven a Dewey de la presidencia quedando, en su lugar el más joven de los tres, Gil Navarro. Dewey continúa como Consejero y promueve una serie de mecanismos legales buscando su restablecimiento.

Los esfuerzos de Dewey tuvieron frutos en diciembre de 2007, por lo que se le tiene que restituir la presidencia. Como reacción a esto, los consejeros Gil y Villarreal publicaron en medios impresos de comunicación una carta abierta en donde se pudo leer que "Los consejeros del ICAI, Manuel Gil Navarro y Alfonso Raúl Villarreal señalamos que Eloy Dewey será Presidente del ICAI en virtud de una resolución jurisdiccional y no del acuerdo ni mucho menos del consenso con sus pares" (Palabra, 12 de diciembre de 2007).

En septiembre de 2008, nueve meses después de que Dewey Castilla reasumir la presidencia, dos de los tres consejeros presentan en sesión y aprueban, sin discusión una nueva plantilla, laboral del instituto. Dewey se manifiesta en contra y sorprendido: el Presidente no podía opinar, siquiera, sobre el organigrama. Es evidente que Villarreal cumplía su palabra de que sería un Presidente por ley, pero no reconocido por sus pares.

Dewey renuncia siendo Gil Navarro quien asume el puesto. El cambio en Presidencia no fue el único, en perspectiva de Alejandro Cárdenas, ex director del ICAI: "después de ese sisma, me parece que se comenzaron a cobrar cuotas de poder. Y empezó a llegar personal vinculado a gobierno.” (Cárdenas, en entrevista). Comienza, también, a cambiar la relación con la sociedad civil organizada, disminuyendo.

\section{CAMBIO DE GOBIERNO: PRIMEROS INDICIOS DE OPACIDAD}

En tanto el Consejo General del ICAI se desgastaba con el conflicto por la Presidencia, aparecieron algunos hechos, en apariencia aislados, pero que en retrospectiva no son sino síntomas de opacidad en la administración de Humberto Moreira Valdés.

Para Carlos Orta, Presidente de la principal fuerza opositora, el Partido Acción Nacional (PAN) y entonces integrante del Poder Legislativo de Coahuila, la interpretación de la oposición sobre el marco normativo vigente es: 
[...] creemos que nuestro Estado contamos con una ley vanguardista en cuanto a acceso a la información pública y protección de datos personales [...] [pero] la administración [de Humberto Moreira Valdés] comienza a encontrar recovecos de cómo sacarle la vuelta a lo que se exige mediante esta ley.

Y agrega:

Vemos que no existe una separación de poderes aquí en Coahuila. Y eso fue lo que nos está trayendo estas consecuencias [lo de la deuda] [...] dejó de tener autonomía e independencia, tanto el poder Legislativo como el poder Judicial. Pero, más aun, organismos que deben ser ciudadanizados fueron cayendo en el control de este gran poder y perdieron sus facultades, su campo de acción y, sobretodo, su autonomía [...] (Orta, en entrevista).

Los síntomas de opacidad se presentan en los programas sociales: algunos medios de comunicación daban a conocerlo y la oposición en el Congreso lo tomaba como tema propio. Se trata de la implementación de programas sociales bajo un esquema nada transparente; y frente a eso, el ICAI no se pronunció.

En el Congreso, la oposición propuso la creación de una Comisión Investigadora y la solicitud de respuesta a una lista de preguntas sobre el programa, mismas que ya se habían presentado ante las oficinas del Ejecutivo Estatal, vía el procedimiento de la Ley de Acceso a la Información, diez meses atrás. Las peticiones contenidas en la propuesta parlamentaria fueron desechadas al no contar con la aprobación de la mayoría. Sobre esto, el balance que hace el Presidente del PAN en Coahuila y Diputado local, Carlos Orta es que:

En Coahuila no sólo se evita que se conozca la información pública: en Coahuila se trabaja, en el gobierno, por tapar la información. No sólo no cumple la Ley [...] sino que procura tapar, ocultar, enterrar mucha otra más [información] para que no sea solicitada. Hay temas que ni siquiera son del conocimiento, no se conoce su existencia; son temas de los que jamás se podrá tener la información (en entrevista).

Un año antes de terminar su periodo como Consejero del ICAI, Manuel Gil dimite y es designado como Consejero del Instituto Electoral y de Participación Ciudadana de Coahuila. Recibe un premio. Alfonso Villarreal asume la Presidencia. Se operaron otros cambios en la normativa relacionada con el ICAI. La más significativa fue la de ampliar el número de Consejeros de tres a cinco. 
Para marzo de 2010, el grupo parlamentario del PAN presenta un punto de acuerdo refiriendo que el Gobierno del Estado no estaba cumpliéndose con la Ley de Acceso a la Información Pública, en particular en lo relativo a los avances de la gestión financiera, la publicación de los contratos celebrados y la entrega de los recursos públicos. Tal y como sucedió el 25 de agosto de 2009, el asunto fue enviado a la Comisión de Finanzas, donde no fue dictaminado. Para la sesión del 10 de agosto de 2010 habían pasado nueve meses de que el grupo parlamentario del PAN había presentado un punto de acuerdo solicitando se hiciere un llamado a las instancias integrantes del Poder Ejecutivo con el fin de que actualizaran la información pública que, por Ley, debía estar en sus páginas. En aquella ocasión, el grupo mayoritario del PRI había argumentado que, a falta de reglamento expedido por el ICAI, estaban imposibilitados para calificar si la información en las páginas estaba o no actualizada por lo que se envió a estudio en comisiones.

Las observaciones hechas por la oposición buscando la actividad del ICAI cobran relevancia porque a la luz del cumplimiento de la Ley, un tema como el de la deuda en Coahuila se hubiese evitado. Cobra también relevancia porque aun cuando reiteradamente se señaló carencias en la actividad del ICAI, la mayoría del PRI en el Congreso ratificó a Alfonso Villarreal como Consejero.

\section{Moreira: Del Gobierno de Coahuila a la dirigencia nacional del PRI}

Diversas modificaciones legales se operaron en Coahuila. La de mayor trascendencia, en retrospectiva, fuela creación del Sistema deAdministración Tributaria de Coahuila (SATEC), que sería una oficina ejecutiva encargada del cobro de contribuciones. Esto fue el 30 de noviembre de 2009. Para entonces, la procuración de justicia había sido también modificada: se creaba la Fiscalía del Estado de Coahuila y se había ampliado la duración de los magistrados del Tribunal de Justicia. El poder se estaba concentrando a partir de reformas a leyes. Particularmente, esto último, la modificación al Poder Judicial traería consecuencias sobre la deuda:

El Poder Judicial sufre una parálisis que se refleja en torno de la deuda [...] presentamos una controversia constitucional local, con el $10 \%$ de la fracción parlamentaria. Lo que no quiso hacer el ICAI, lo hicimos nosotros [los del PAN], y hasta este momento, setenta u ochenta días después de que fue presentada, no hemos tenido un acuerdo de trámite (Flores Hurtado, en entrevista).

Concentrado el poder, el 3 de enero de 2011, ante el Pleno del Congreso, 
Humberto Moreira Valdés solicita licencia para separarse de su cargo a partir del siguiente día y hasta el 30 de noviembre, día en que terminaría su periodo constitucional. Tres diputados hablaron en tribuna: dos en contra y uno a favor. En contra, Esther Quintana Salinas destaca:

Muchos gobiernos hoy día no son por sí mismos ni exitosos o fallidos, eficientes o ineficientes, son como la mercadotecnia los inventa y los vende, de ahí las cantidades millonarias del presupuesto público que se destinan a la creación de una imagen, al posicionamiento de un personaje político, a la entronización de una persona a la que le fabrican una leyenda, una historia en la que se sobre valoran sus atributos, se le adjudican los que no tiene y se minimizan o de plano se eclipsan sus defectos o debilidades [...] No se vale decir, ahí nos vemos, cuando el estado que el señor gobierna tiene una deuda pública que se minimiza, alegando que es de esas proporciones, casi 8 mil millones, porque el estado es el aval de las que han adquirido los municipios y sabrá Dios, porque de eso no tenemos noticia ni siquiera aquí en el órgano legislativo de la que se tiene con proveedores y contratistas, y con las reformas aprobadas la semana pasada por la mayoría de este Congreso, van por más deuda que van a pagar ¿quiénes? los coahuilenses [...] Y esto nos lleva al señalamiento de otra ausencia: la transparencia, que en Coahuila no constituye prioridad para un gobierno que se ostenta como democrático intra y extra muros, la opacidad la tenemos oficializada. En las páginas de las dependencias estatales se publica lo que se quiere, como se quiere y cuando se quiere, verbigracia y las reglas de operación de los programas sociales y sus beneficiarios son parte del capítulo de los misterios sin resolver [...] 107 millones de pesos (unos US \$ 8.900.000) cuesta (sic) a los coahuilenses el ICAI ¿para qué?, triste papel, si vivimos en el oscurantismo, no es cierto que Coahuila sea la sucursal del paraíso terrenal, qué más quisiéramos [...] (Congreso de Coahuila, Diario de Debates, 3 de enero de 2011).

\section{Lo QUe CINCO AŃos FUe UN SECRETO: LA DEUdA PÚblica EN COAHUILA}

En entrevista concedida al Grupo Reforma, el Auditor Superior del Estado de Coahuila reconocería en diciembre de 2011 que el monto de los créditos que tuvo el Estado sumaban al menos US \$2.050.000.000, de los cuales sólo una tercera parte estaban registrados ante la autoridad federal competente (la Secretaría de Hacienda y Crédito Público). Esa cantidad fue negada o desconocida por Humberto Moreira Valdés, quien fuera gobernador del Estado en el momento que se presentan las irregularidades: aun cuando se incumplía la Ley de Acceso a la Información Pública en cuanto a la publicación de la información mínima, aun cuando se incumplía la Ley 
de Deuda que obligaba a hacer pública la información de los créditos contratados, aun cuando la oposición reiteradamente solicitaba se aclarara la situación, el ICAI guardó silencio.

El 25 de agosto de 2009 por primera vez se presenta, de parte de la oposición, el tema de la deuda. Se hace a través de una proposición de punto de acuerdo que fue turnado a la Comisión de Finanzas. El 3 de agosto del 2010, once meses antes de que se conociera el tema de la deuda, por primera vez se somete a consideración un punto de acuerdo "de urgente y obvia resolución” relacionado con la deuda. El grupo parlamentario del PAN insiste en la ausencia de información refiriendo fuentes del Gobierno Federal, según información de la Secretaría de Hacienda y Crédito Público Coahuila ocupa el primer lugar en incremento de Deuda Pública entre todas las entidades federativas aumentando en un año, más de un $280 \%$.

\section{Y solicita,}

[...] respetuosamente al Ejecutivo del Estado, a través de la Secretaría de Finanzas, se informe a esta Soberanía el monto de la deuda pública actual, los rubros en los que se ha ejercido y la proyección que se tiene para el término de la administración.

Por decisión mayoritaria, el asunto no se resolvió en la Asamblea y fue turnado a la Comisión de Hacienda y Cuenta Pública, donde nunca fue dictaminado.

El 12 de octubre de 2010, la oposición insiste en el tema de la deuda. Proponen que el Congreso, por conducto de la Comisión de Hacienda y Cuenta Pública, solicite a la Secretaría de Finanzas del Estado un informe sobre la situación de la Deuda Pública de la entidad. Por decisión mayoritaria, el asunto no se resuelve en la Asamblea y es turnado para su estudio a Comisión y nunca se dictamina. El 1 de febrero de 2011, el grupo parlamentario del PAN presenta una proposición con punto de acuerdo por el que se solicitaría al jefe del Ejecutivo del Estado de Coahuila un informe detallado sobre los créditos que ha solicitado el gobierno estatal durante el periodo comprendido del año 2007 a esa fecha. La propuesta, sin embargo fue retirada del orden del día por solicitud de los autores y ya no se presenta en la siguiente sesión. El 25 de marzo de 2011, el grupo parlamentario del PAN propone se acuerde solicitarles al Secretario Ejecutivo del SATEC, Javier Villarreal Hernández, y al Tesorero General del Estado, Jesús Ochoa Galindo, un informe sobre el monto total de la Deuda Pública del Estado, en los rubros a corto y largo plazo. En esta ocasión el monto global de la deuda se estima cerca de US \$ 1.500.000.000 y no, como hasta entonces se decía, de poco más de US \$ 650.000.000. 
En julio del 2011, hubo elecciones en Coahuila. Las posiciones en disputa electoral fueron las de Gobernador del Estado y diputados locales. Una semana antes de la jornada electoral, el grupo parlamentario del PAN en el Congreso del Estado hizo del conocimiento público y, después, denunció ante las autoridades irregularidades en la contratación de deuda pública. Las acciones ilegales alrededor de la deuda transcurren, al menos, por dos caminos diferentes: 1) la falsificación, de parte de funcionarios del Gobierno del Estado de Coahuila, de periódicos oficiales en los que se pretendió hacer pasar como existentes Decretos del Congreso por los que se habría autorizado la contratación de deuda; 2) la falsificación de documentos al interior de la Secretaría de Hacienda y Crédito Público (SHCP). Esto no incluye las validaciones, a través de la abrogación de la Ley de Deuda, que hiciere en agosto de 2011 el Congreso del Estado, ni la explicación del enriquecimiento inexplicable a favor de, al menos, colaboradores cercanos a Humberto Moreira Valdés y la ausencia de explicación sobre en qué se utilizó el dinero que se debe. Tampoco incluye obra pública realizada y no pagada equivalente a una suma millonaria que no se ha especificado (Reforma, 7 de diciembre de 2011).

Siendo ya del dominio público la contratación ilegal de parte de la deuda y existiendo acciones de parte del Ejecutivo y la mayoría en el Legislativo para que el monto de la misma no se conociera, habría de sumarse la información que comenzó a conocerse sobre la acumulación de recursos de personas cercanas al gobernador Humberto Moreira Valdés, que no podían, no pueden, justificarse legalmente.

Sobre todo este asunto, el ICAI no se pronunció. ¿Tendría que hacerlo? De haberse hecho cumplir la Ley de Acceso a la Información Pública, en la parte relativa a la información de oficio, todos los movimientos presupuestales hubieran podido verse. Con toda esta información en conocimiento de la opinión pública, con procedimientos iniciados ante las autoridades competentes y ante la ausencia de una posición clara de parte de la autoridad constitucional en la materia de transparencia, el tema de la deuda seguía en Coahuila.

¿Cómo germinó el tema de la deuda con la segunda mejor ley en materia de acceso a la información? Desde la perspectiva del abogado de un grupo de sociedad civil que impugna la deuda en Coahuila,

[...] no existe el cumplimiento de la norma [de Acceso a la Información] que los obligue a tener una información mínima y que el Instituto [Coahuilense de Acceso a la Información] jamás los ha requerido en ese sentido [...] hubo muchas omisiones, pero el camino que ha seguido el Estado para ir legalizando todo este tipo 
de latrocinios tiene que ver con muchas complicidades: y una de esas es un instituto que no ha cumplido con su función. (Flores Hurtado, en entrevista).

\section{Y agrega:}

[Sobre la deuda] Lo menos grave es el monto, a pesar de lo escandaloso. Lo más grave es la manera en la que actuó el gobierno, en complicidad con el Congreso y otros órganos de gobierno para proteger a los culpables [...] [para contextualizar] la ley de deuda pública o bajo la cual se contrató la deuda de Coahuila [...] [prohibía] contratar deuda de corto plazo por un monto superior al $5 \%$ del presupuesto anual: hoy la deuda oscila en el orden del 120\% del presupuesto anual. Es decir, había violado el tope de la Ley por mucho. Y esa Ley, no les permitía, además, lavarle las manos a los culpables de la deuda [...] abrogan la Ley y crean una nueva Ley a modo donde le quitan los candados para poder reestructurar y refinanciar la deuda [...] y lo hacen, expresamente, con efectos retroactivos: [en la exposición de motivos dice] "esta Ley será aplicable para los créditos aprobados con anterioridad de su vigencia a corto plazo" [...] y dicen que la presente Ley tendrá, por objeto [...] permitir la reestructura o financiamiento de créditos aun, cuando por alguna causa, se hubieran contratado sin cumplir con los requisitos legales (Flores Hurtado, en entrevista).

El cumplimiento a cabalidad de la Ley de Acceso a la Información hubiese inhibido lo que después se convirtió en un problema agudo. Considerando que la información financiera era lo que mínimamente la Ley en la materia exigía debiera publicarse ¿pudo evitarse el vacío de información en torno de la deuda?:

Desde luego, la publicación de los estados financieros es una obligación que establece el artículo 19. Esa obligación se vino dando hasta el 2008. Sin embargo, si tú ves, en el Poder Ejecutivo nunca se le ha calificado con un cien. ¿Por qué? Porque uno de los puntos que faltan (Villarreal, en entrevista).

En el ICAI sabían que el Ejecutivo no cumplía con la Ley y, sin embargo, no hicieron algo para obligarlos a cumplirla aun cuando tenían las facultades legales para hacerlo.

Integrantes de la sociedad civil consideran que la falta de voluntad de quienes integran el ICAI para hacer que el Ejecutivo cumpliera con la Ley se debe a una subordinación de hecho. 
Desde afuera del ICAI, la evidencia sugiere omisiones. El haber hecho cumplir la Ley de Acceso a la Información Pública muy probablemente hubiese inhibido la corrupción presentada en el sexenio de Humberto Moreira Valdés y el tema de la deuda así como el desconocimiento que hasta ahora existe sobre el destino verdadero de más de US \$3.000.000.000 (a febrero de 2012), se conocería. Desde adentro del ICAI, sin embargo, la percepción que tienen o que sostienen tener es que el trabajo se ha cumplido a cabalidad: "Yo creo que el trabajo realizado en siete años debe ser debidamente ponderado", dice el Director General, Miguel Ángel Medina (en entrevista).

\section{EN EL ICAI: EL OLVIDO DEL COMPROMISO PÚBLICO Y LA UTILIZACIÓN DE LA ORGANIZACIÓN PARA FINES PRIVADOS}

Mientras que todo lo de la deuda sucedía en Coahuila, el ICAI omitió asumir alguna postura. Mantuvo su trabajo regular y, en términos generales, se aprovechó de la organización pública para la satisfacción de intereses particulares de quienes la integran. Existen en la Ley del ICAI algunos mecanismos que hubieran podido incidir en su pasividad, pero nunca se han llevado a la práctica.

El ICAI tampoco reforzó su Programa de Publicidad y Propaganda. Después de que Alfonso Villarreal Barrera asumiera la presidencia del Consejo General del ICAI (esto en 2009), el presupuesto ejercido en ese rubro cayó a la tercera parte de lo que venía invirtiéndose en este rubro. El ex Director General del ICAI, Alejandro Cárdenas, comenta: "es evidente que [después de lo que viví], se redujo el presupuesto en publicidad. $\mathrm{O}$ no sé si no se redujo, pero no se ve: no lo tengo claro" (Cárdenas, en entrevista). Por su parte, Alfonso Villarreal, Presidente del ICAI reconoce:

Sí hay un cambio de estrategia [sobre la propaganda] [...] nosotros, los primeros años, hicimos una campaña, en términos políticoselectorales, una campańa de aire para dar a conocer el tema y para dar a conocer el instituto. Y luego, decidimos cambiar a una campaña de tierra. Porque quisimos apostarle a las instituciones de educación y, específicamente, a las de educación media superior [...] son las prepas y las técnicas (Villarreal, en entrevista).

Esta actitud, sin embargo es inconsistente con la información que el propio ICAI tenía: en julio de 2008 contrató a la firma Berumen para elaborar un estudio de opinión relacionado con la transparencia en Coahuila del cual se conoce que de 2006 a 2008 se incrementó el porcentaje de personas que no conocían el procedimiento para solicitar 
información ${ }^{8}$. Es decir, aun cuando de 2006 a 2008 el desconocimiento de algo básico como el procedimiento para ejercer el derecho de acceso a la información había crecido 20 puntos porcentuales, el ICAI decidió recortar a una tercera parte el presupuesto ejercido en propaganda y publicidad. A la Secretaría Técnica le asignaron asesores en cuyo contrato se acordaba el desarrollo y actualización del marco jurídico y reglamentos: por más de cinco años, no hubo en el ICAI actualización de reglamento alguno. En el rubro presupuestal de profesionalización, se han pagado cursos de inglés, presentación de exámenes TOEFL y presentación de exámenes de ubicación en este idioma (sin que el inglés tenga clara relación con su trabajo). En 2010 y 2011, el Presidente y el Director General se inscribieron en un curso de Eneagrama. Al Director Jurídico se le pagó un Diplomado en Derecho Electoral.

En 2011, el año en que el tema de la deuda estaba en su momento más álgido y cuando los señalamientos sobre la ausencia de transparencia en el ejercicio del gasto era denuncia pública en medios de comunicación, el ICAI certificó algunas áreas bajo normas de la Organización Internacional para la Estandarización. En el apogeo del problema de la deuda, agrupaciones ciudadanas acudieron al ICAI para solicitarle por escrito se pronunciara al respecto: "Como le decía yo al Presidente del ICAI: 'si ustedes, que son el órgano ciudadano encargado de defender la transparencia no se manifiestan en el tema, entonces ¿qué es lo que vamos a hacer?'”, dice en entrevista Flores Hurtado. Y agrega:

Uno de los detalles que quisimos entender fue ver cómo reaccionaban los organismos de transparencia en torno a la deuda [...] [específicamente] los organismos ciudadanos. Sabemos que en Coahuila no hay división de poderes: el Congreso está supeditado al capricho del gobernador, también el Poder Judicial del Estado (Flores Hurtado, en entrevista).

¿Qué se esperaba del ICAI? El mismo entrevistado, Reyes Flores Hurtado, lo explica:

El ICAI tiene la facultad para promover la controversia constitucional contra todo acto que, en la materia de su competencia que es la transparencia, sea directamente violatorio de un principio constitucional. Bajo esta lógica, nosotros le pedimos al ICAI que promoviera una controversia constitucional en contra del decreto

8 Mientras que en 2006, en un estudio similar también considerado por el ICAI a la pregunta ¿ Sabe usted cuál es el procedimiento para solicitar información a los organismos públicos de Coahuila? El 52.4\% contestó "No sabe”, en 2008, a la misma pregunta, el $72.4 \%$ contestó "No sabe". 
que aprueba la deuda [...] bajo el argumento de que había una violación al principio de transparencia. Y por supuesto que lo había: el Congreso aprueba la deuda de manera inusitada, sin tener un solo documento que justifique el origen del crédito. O sea, no tuvo [el Congreso] ni un solo contrato de crédito para decir que la deuda era legal. Pero, además, ellos [los del Congreso] sabían que la deuda se había contratado violando la Ley, porque ellos sabían que debían de haber aprobado la contratación de la deuda y no lo habían hecho, no al menos por esos montos. Tampoco tuvieron a disposición los documentos que justificara el destino del recurso, que era parte de lo que tuvieron que haber analizado: por qué, porque el Congreso no puede solapar un desvío o un lavado de dinero, por ejemplo. Y le pudieron haber metido dinero lavado y no se hubiera dado cuenta porque no analizó ningún documento. Desde esta lógica, desde nuestro punto de vista, el ICAI debió haber intervenido presentando una controversia porque había una violación a la materia de su competencia. Lo menos que debió hacer el gobierno del Estado era poner [a disposición] la documentación al Congreso. Y lo menos que pudo haber hecho el Congreso fue exigirla para saber qué era lo que iba a aprobar [...] bueno, el ICAI se niega a presentar la controversia, bajo el argumento de que no está en su competencia controvertir esos actos (Flores Hurtado, en entrevista).

Desde la perspectiva del ICAI, de su Presidente, lo que el grupo ciudadano pedía no podía realizarse: "les dimos [a los de Claridad y Transparencia], en tiempo y forma, una respuesta donde nosotros les argumentamos jurídicamente que no éramos competentes en ese tema, respecto a la acción de inconstitucionalidad" (Villarreal, en entrevista).

\section{HALLAZGOS DEL CASO: A MANERA DE CONCLUSIÓN}

En Coahuila, la transparencia se inicia como una política pública que tiene el apoyo de todos los poderes del Estado: como en ningún otro gobierno subnacional, en Coahuila el tema comienza con la firma de un convenio interinstitucional entre el Ejecutivo, el Legislativo, el Judicial y el Instituto Electoral. Entre los compromisos asumidos se encontraba el de realizar una serie de foros de participación ciudadana y consulta de los que se obtendría el insumo para la redacción de la reforma constitucional pertinente y tres leyes relacionadas con la transparencia. Es, como puede verse, una política que inició con una clara muestra de voluntad política. Como sucedería en el estudio seminal Pressman y Wildawsky (1998), la experiencia en Coahuila impulsa a la búsqueda de una explicación pues se ha malogrado una política que desde su comienzo gozó de consenso serio, disponibilidad de recursos, criterios legislativos precisos y compatibles y la oposición fue 
inexistente. Y más allá: el organismo encargado de la implementación, el ICAI estaría legislativamente diseńado para que contara con las mayores fortalezas posibles, entre ellas el de ser un organismo constitucional autónomo. Con este prometedor inicio, cabe la pregunta de Luis F. Aguilar (2000) (ya destacada en la primera parte de este estudio): ¿por qué buenas leyes, inteligentes programas gubernamentales y oportunos financiamientos, esfuerzos cotidianos de organizaciones y personas, simplemente no funcionaron, no resolvieron los problemas sociales que afrontaron y quedaron muy lejos de los objetivos que prometieron alcanzar?

La brecha de la implementación se presentó por situaciones de carácter político, por acuerdos entre grupos, por la concentración del poder en unas pocas manos. De esto no se encuentra en la literatura fuera de la realidad latinoamericana.

¿Qué sucedió a nivel organizacional en el ICAI? Una primera generación de consejeros (titulares del órgano superior de toma de decisiones) se contaminó en una lucha por ocupar la presidencia del organismo. Originalmente, la Ley establecía que quien fuere nombrado Presidente lo sería por el tiempo que fuera Consejero. El parecer de quienes integraron la primera generación fue contraria a lo establecido por la norma: se intentó un acuerdo para acortar artificialmente la presidencia, vía la renuncia del encargo de quien fuera el primer Presidente, para que los otros consejeros pudieran ocupar el puesto. Esto se identifica plenamente con las distorsiones burocráticas, aludidas por Aguilar Villanueva (2000)

A lo largo de su existencia, posterior al primer reacomodo en el Consejo General significando la salida de la organización de su primer Presidente y que otro de los consejeros asumiera la titularidad de una consejería en el órgano Electoral, la Ley del ICAI se reformó para permitir la reelección de los Consejeros a través de un filtro político: el Congreso del Estado. En este sentido, el único que ha sido premiado con la reelección ha sido el Presidente quien ha dejado, a su paso, más pendientes que logros.

Dos son los mensajes claros que pueden derivarse de la experiencia en Coahuila: el primero, que la Ley por sí misma no es requisito suficiente para garantizar la implementación exitosa de una política pública; la segunda, que la organización pública, instrumento para la implementación, no se encuentra aislada de su entorno.

La discusión sobre la ausencia de transparencia en el manejo financiero en el Gobierno del Humberto Moreira Valdés se presentó en el Congreso del Estado: el ICAI, aun siendo el órgano garante, no asumió posicionamiento alguno aun cuando, aceptado en las entrevistas sostenidas 
para este trabajo, se ha reconocido que de haberse hecho cumplir la Ley de Acceso a la Información Pública y Protección de Datos Personales vigente, hubiese sido posible aminorar los probables actos de corrupción que se presentaron. Todo intento de parte de la magra oposición en el Congreso de Coahuila por pedir una actitud activa y propositiva al ICAI, que ante evidentes faltas a la Ley en la materia, asumiera una postura, la mayoría en el Congreso, fue obstaculizado por la mayoría. En los tres ańos de la LVIII Legislatura, en la cual se presenta el problema de la deuda pública, no existió argumento alguno de parte de la mayoría para no aceptar las propuestas de la minoría.

Mientras el problema iba creciendo, así como fueron creciendo las denuncias de falta de transparencia, la actitud de la organización fue de indiferencia ante el problema. Más allá de la indiferencia, la evidencia sugiere el aprovechamiento para fines privados de los recursos de la organización pública.

El estudio de un caso instrumental sobre implementación de políticas públicas en un contexto latinoamericano nos enfrenta ante el factor político, mismo que no es claro en los estudios desarrollados en contextos americanos o europeos. 


\section{REFERENCIAS}

Aguilar Villanueva, L. F. (Comp.). (1993). Problemas Públicos y Agenda de Gobierno. Mexico: Miguel Ảngel Porrúa.

(Comp.). (1996). La Hechura de las Politicas Publicas. México: Miguel Ángel Porrúa.

(2000). Estudio Introductorio. En Aguilar Villanueva, L. F. (Comp.), La Implementación de las Politicas Publicas. México: Miguel Ángel Porrúa.

Bastien, J. (2009). Goal Ambiguity and Informal Discretion in the Implementation of Public Policies: The Case of Spanish Immigration Policy. International Review of Administrative Sciences, 75 (4), 665-685.

Bergman, M. y Lane, J. (1990). Public Policy in a Principal-Agent Framework. Journal of Theoretical Politics, 2 (3), 339352.

Berman, P. (2000). El Estudio de la Macro y la Micro-Implementación. En Aguilar Villanueva, L. F. (Comp.), La Implementación de las Políticas Publicas. México: Miguel Ángel Porrúa.

Cairney, P. (2009). Implementation and the Governance Problem: A Pressure Participant Perspective. Public Policy and Administration, 24 (4), 355-377.

Centro de Investigación y Docencia Económicas (CIDE). (2010). Métrica de la Transparencia 2010. México: CIDE.

Comfort, L. K. (1981). Goals and Means: The Problem of Specification in the Development of Effective Public Policy. Administration \& Society, 13 (1), 77-108.

Cooley, V. (2010). Community-Based Sanctions for Juvenile Offenders: Issues in Policy Implementation. Criminal Justice Policy Review, 22 (1), 65-89.

Derthick, M. (1972). New Towns In-Town. Washington: The Urban Institute.

Elmore, R. (2000) Diseño Retrospectivo: La Investigación de la Implementación y las Decisiones Políticas. En Aguilar Villanueva, L. F. (Comp.), La Implementación de las Políticas Publicas. México: Miguel Ángel Porrúa. 
Goggin, M. (1986). The “Too Few Cases/Too Many Variables" Problems in Implementation Research. Political Research Quarterly, 39 (2), 328347.

Goodin, R. E. (2003). Las Instituciones y su Diseño. En Goodin, R. E. (Comp.), Teoría del Diseño Institucional. Barcelona: Editorial Gedisa.

Grindle, M. S. (2009). La Brecha de la Implementación. En Mariñez Navarro, F.y Garza Cantú V. (Eds.), Política Pública y Democracia en América Latina. Del Análisis a la Implementación. México: Miguél Ángel Porrúa-EGAP-CERALE.

Hasenfeld, Y. y Brock, T. (1991). Implementation of Social Policy Revisited. Administration \& Society, 22 (4), 451-479.

Hepburn, J. y Goodstein, L. (1986). Organizational Imperatives and Sentencing Reform Implementation: The impact of Prison Practices and Priorities on the Aattainment of the Objective of Determinate Sentencing. Crime \& Delinquency, 32 (3), 339-365.

McLaughlin, M. W. (1987). Learning from Experience: Lessons from Policy Implementation. Educational Evaluation and Policy Analysis, 9 (2), 171-178.

Myrtle, R. C. (1983). A Managerial View of Policy Implementation. The American Review of Public Administration, 17 (1), 17-32.

Parsons, W. (2007). Politicas Públicas. México: FLACSO-Miño y Dávila.

Pressman, J. L. y Wildavsky, A. (1998). Implementación. Cómo grandes expectativas concebidas en Washington se frustran en Oakland. México: Colegio Nacional de Cienias Políticas y Administración Pública A.C.Fondo de Cultura Económica.

Rein, M. y Rabinovitz, F. F. (2000). La Implementación: Una Perspectiva Teórica. Entre la Intención y la Acción. En Aguilar Villanueva, L. F. (Comp.), La Implementación de las Políticas Publicas. México: Miguel Angel Porrúa.

Sabatier, P. A. y Mazmanian, D. A. (2000). La Implementación de la Política Pública: Un Marco de Análisis. En Aguilar Villanueva, L. F. (Comp.), La Implementación de las Politicas Publicas. México: Miguel Ángel Porrúa.

Sarbaugh-Thompson, M. y Zald, M. N. (1995). Child Labor Laws: A His- 
torical Case Of Public Policy Implementation. Administration \& Society, 27 (1), 25-53.

Van Meter, D. S. y Van Horn, C. E. (2000). El Proceso de Implementación de las Políticas. Un Marco Conceptual. En Aguilar Villanueva, L. F. (Comp.), La Implementación de las Politicas Publicas. México: Miguel Angel Porrúa.

\section{Documentos oficiales}

Congreso de Coahuila, Diario de Debates, 2010-2011.

\section{Periódicos}

Palabra, Saltillo, Coahuila, México, 2007

Reforma, México, DF, México, 2011.

\section{Entrevistas}

Alejandro Cárdenas López, Director General del ICAI, entrevistado el 26 de noviembre de 2011.

Eloy Dewey, Ex Presidente del ICAI, entrevistado el 9 de noviembre 2011.

Jesús Flores Mier, Consejero del ICAI, entrevistado el 20 de diciembre de 2011.

Reyes Flores Hurtado, Representante Legal de Claridad y Transparencia A.C y activista de Brigada Jurídica en Contra de la Deuda del Estado de Coahuila, ex-dirigente estatal del PAN, entrevistado el 20 de diciembre de 2011.

Luis González Briseño. Consejero del ICAI, entrevistado el 21 de diciembre de 2011.

Teresa Guajardo Berlanga, Consejera del ICAI, entrevistada el 4 de enero de 2012.

Nelly Herrera, Alianza Cívica de Coahuila, entrevistada el 16 de noviembre de 2011.

Miguel Ángel Medina Torres, Director General del ICAI, entrevistado el 21 de diciembre de 2011.

Carlos Orta Canales, Dirigente del Partido Acción Nacional en Coahuila y Diputado Local 2009-2011, entrevistado el 20 de diciembre de 2011. 
Gloria Tobón de Garza, Representante de Asociación de Usuarios de Aguas de Saltillo (AUAS), entrevistada el 5 de diciembre de 2011.

Alfonso Villarreal Barrera, Consejero Presidente del ICAI, entrevistado el 20 de diciembre 2011.

Recibido: 22-04-2013

Aceptación de la versión final: 22-11-2013 